\title{
And now we are five
}

\author{
Nature Astronomy turned five this month. We share some of our most popular covers and impactful papers, and \\ look forward to more discoveries in 2022.
}

( n 4 January 2017, Nature Astronomy launched with a mission to publish significant advances in astronomy, astrophysics and planetary sciences, including their societal impact. We have since published five full volumes, which constitute 1,420 articles spanning opinion pieces, progress in research and reviews - plus Corrections and Matters Arisings, which are important venues for maintaining a correct and transparent record. We always seek to place discoveries and developments in their proper context, with lucid writing, clear display items and detailed methodology and supplementary data. This endeavour involves authors, reviewers, manuscript editors, copy editors and art editors.

Besides working on figures and tables, the art editors also produce our monthly covers. The style has evolved subtly, but we always start with a stunning image. Figure 1 shows the most popular covers for each year - chosen by the editors for the first year, and subsequently by our Twitter followers. These are, from left to right: a drawing of the Cassini explorer by James Tuttle Keane in celebration of the completion of the Cassini-Huygens mission, for which we commissioned a number of News \& Views pieces from its fond users on their favourite images; a closeup of one of the telescopes of the Australian Square Kilometre Array Pathfinder that accompanied our Focus on fast radio bursts; a historical take on the time series of the sunspot number over 400 years; a SOFIA far-infrared image of the collapse of magnetized gas within the interstellar medium; and finally, a glorious composite image of Centaurus A from SOFIA, the Atacama Pathfinder Experiment, Chandra X-ray Observatory and MPG/ESO 2.2-m telescope that accompanied a study of the molecular warped disk of Cen A.

As one shouldn't judge a journal by its cover, this month we are also showcasing (with free access) six different articles that reflect the essence of Nature Astronomy. The scientific papers were selected for both their novelty and impact within their research fields.

'A seven-planet resonant chain in TRAPPIST-1' by Rodrigo Luger et al. investigated the complex three-body resonances linking every member of this planetary system. When we received the paper, the discovery of the planetary septuplet was still fresh, and this study was the first to use the latest $\mathrm{K} 2$ data to constrain its architecture. TRAPPIST-1 remains significant because it has at least seven planets of similar radius to Earth, with three or four of them lying in the radial zone that could support liquid water.

'Quantitative evaluation of gender bias in astronomical publications from citation counts' by Neven Caplar, Sandro Tacchella and Simon Birrer used machine learning techniques to quantify the gender bias in astronomy publishing, robustly showing that papers led by women have $10 \%$ systematically fewer citations than those authored by men. This paper is part of our Focus on gender equity in astronomy, which explores the often subconscious biases and discriminatory behaviours experienced by women (and minority astronomers) in almost every aspect of scientific discourse (from conferences to missions, career prizes and citation counts). However, there are signs of improvement from collective action at the individual, institutional and international level, as spotlighted in our follow-up Focus on Diversity, Equity and Inclusion best practices and solutions.

'Relativistic Shapiro delay measurements of an extremely massive millisecond pulsar' by $\mathrm{H}$. Thankful Cromartie et al. reports probably the most massive neutron star discovered so far by a combination of NANOGrav 12.5-yr data with radio data from the Green Bank Telescope. Millisecond pulsar J0740+6620 has a mass of $2.14 M_{\odot}, \sim 0.1 M_{\odot}$ more massive than the previous record holder, very close to the upper limit on neutron star masses from LIGO measurements.

"Tensions between the early and late Universe' by Licia Verde, Tommaso Treu and Adam Riess is a Meeting Report on a workshop at the Kavli Institute for Theoretical Physics in July 2019, focusing on the discrepancy in measurements/ calculations of the Hubble constant between probes of the early Universe (the physical
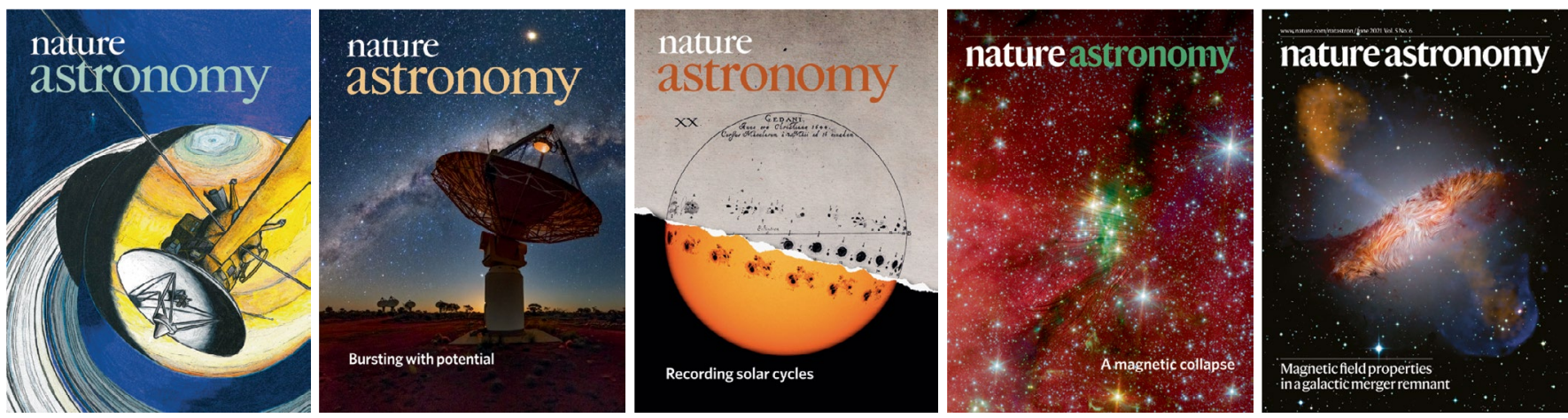

Fig. 1 | Our most popular covers from 2017 to 2021. From left to right: the Cassini explorer (image: James Tuttle Keane, California Institute of Technology; cover design: Alex Wing), ASKAP array (image: Alex Cherney; cover design: Allen Beattie), the Sun (image: World History Archive / Alamy Stock Photo (top), courtesy of NASA/SDO and the AIA, EVE, and HMI science teams (bottom); cover design: Allen Beattie, Andrés Muñoz-Jaramillo and José M. Vaquero), the interstellar medium (image: NASA/SOFIA/T. Pillai/J. Kauffmann, NASA/JPL-Caltech/L. Allen; cover design: Bethany Vukomanovic) and Centaurus A (image: Enrique Lopez-Rodriguez; cover design: Bethany Vukomanovic). 
size of the sound horizon used to interpret the cosmic microwave background and baryon acoustic oscillations) and those of the local, late-time Universe. Solving the issue requires "a coordinated effort from the sides of theory, interpretation, data analysis and observations", and the article ends with a guide to best practices to "streamline the interaction between these different communities and promote the transparent transfer of information". Later that year, half the Nobel Prize in Physics would go to James Peebles for his foundational work on the $\Lambda$ cold dark matter model. A Collection of papers from the Nature Portfolio on cosmology (and exoplanets) marks this occasion.

'The carbon footprint of large astronomy meetings' by Leonard Burtscher et al. exemplifies our initial considerations of the impact of astronomy on the climate, a pressing topic in our community for the coming decade. COVID-19 prompted meetings to be held online, and this Comment showed that the carbon footprint of the virtual European Astronomical Society meeting of 2020 was roughly 3,000 times smaller than that of the face-to-face one in 2019. In the same issue we also published other Comments on greenhouse gas emissions of the Max Planck Institute for Astronomy in Heidelberg and their proposed measures for reductions; on carbon emissions of the Canada-FranceHawaii Telescope, with a per employee emission that is six times above the recommendation of the Paris Agreement; on the ecological impact of the use of (super) computing and best practices; and on the impact of climate change itself on the quality of astronomical observations. We recently followed up with three further Comments on what astronomers can do, in terms of making astronomy sustainable, communicating climate change effectively and joining forces with other advocacy groups for the climate emergency.

'Phosphine gas in the cloud decks of Venus' by Jane Greaves et al. attracted immediate attention and intense scrutiny, with phosphine being a potential biosignature. Its presence on Venus is mysterious, as the atmosphere should not be able to sustain it - meaning, either our understanding of phosphine chemistry is incomplete or there is a source on Venus. Despite the careful wording, all attention was focused on the latter, and headlines screamed out that life had been found on our nearest neighbour. Soon after publication, we received a Matters Arising questioning the results and methodology. It arrived as the authors were awaiting a recalibration of the ALMA data, which eventually reduced the strength of the phosphine detection. We then published a Reply to the Matters Arising as well as an Addendum. The story exemplifies our commitment to maintaining an objective scientific record. An Editorial sets out the details and contextual framework of the paper. The phosphine question remains open, perhaps to be answered by the three future missions to Venus to be launched around 2030.

In the meantime, there are many things to look forward to this year. Also in this issue, we have a News Feature on the James Webb Space Telescope and the origins of life. Since its exciting Christmas Day launch, everything has been going according to plan, so we hope to start seeing some Early Release Science results soon. The issue also includes our first Research Briefing on 'Polarimetric detection of non-radial oscillation modes in the $\beta$ Cephei star $\beta$ Crucis', which brings together commentary from the authors, one of the reviewers and the handling editor for a multifaceted summary of the paper and its implications. We have a lot more in store for 2022, not least more contributions to our Collection on dwarf galaxies and additional voices to join our Focus on astronomy for development.

Published online: 21 January 2022 https://doi.org/10.1038/s41550-022-01602-5 